M. U. Bhutta, et al., Int. J. Comp. Meth. and Exp. Meas., Vol. 7, No. 3 (2019) 226-235

\title{
NOVEL EXPERIMENTAL SETUP TO ASSESS SURFACES IN TRIBO-CONTACT LUBRICATED BY THE NEXT GENERATION OF ENVIRONMENTALLY FRIENDLY THERMOFLUIDS
}

\author{
MUHAMMAD USMAN BHUTTA ${ }^{1,2}$, ZULFIQAR AHMAD KHAN $^{1} \&$ NIGEL GARLAND $^{1}$ \\ ${ }^{1}$ Faculty of Science \& Technology, Department of Design \& Engineering, NanoCorr, Energy \& Modelling \\ (NCEM) Research Group, Bournemouth University, Talbot Campus, Fern Barrow, Poole, BH12 5BB. \\ ${ }^{2}$ School of Mechanical \& Manufacturing Engineering (SMME), National University of Sciences \& Technology \\ (NUST), Campus H-12, Islamabad, Pakistan.
}

\begin{abstract}
Environmental concerns related to global warming and ozone depletion triggered the introduction of the fourth generation of thermofluids. Amongst the recently introduced thermofluids, one of the most promising fourth generation of thermofluids are hydrofluoroethers (HFEs). HFEs have zero ozone depletion potential and have a lower global warming potential as compared to widely used thermofluids. The type of thermofluid used in a thermodynamic cycle directly affects the tribological performance of the system. HFEs have been reported to have good thermodynamic properties. The overall tribological performance of HFEs however have to be investigated in detail in order to fully assess the mechanical behaviour of interacting components utilizing these thermofluids. This study is concerned with the experimental test rig design modifications and commissioning to conduct tribological testing with HFEs as lubricants. This article covers the experimental test rig design and setup. Experiments to analyse the frictional force, the coefficient of friction and wear by using Hydrofluororther-7000 (HFE-347mcc3) as lubrication medium have been conducted. Industrial applications were simulated by varying test conditions and the results are presented in this paper.

Keywords: bench testing, environment-friendly thermofluids, friction, refrigerants, tribology, wear.
\end{abstract}

\section{INTRODUCTION}

Increase in global population, rise in worldwide economic development and the anthropogenic global climate change have considerably increased the use of cooling, refrigeration and air-conditioning systems worldwide. The selection of a refrigerant depends on the place of use and its application. Some of the refrigerants being used today in the domestic and commercial sector such as ammonia, carbon dioxide and various hydrocarbons are naturally occurring whereas majority of the thermofluids have been artificially formulated.

The first artificially formulated commercial refrigerants were chlorofluorocarbons (CFCs) and hydrochlorofluorocarbons (HCFCs) which were introduced in the 1930s [1]. CFCs and HCFCs proved to possess excellent thermodynamic, heat transfer and tribological properties especially when used in domestic, commercial and small-scale industrial applications [1]. CFCs and HCFCs showed extreme pressure effect and resulted in the formation of protective surface films on the operating machine parts which helped in reducing friction and wear [2-4]. However, the destructive effects of CFCs and HCFCs on the ozone layer were discovered and Montreal Protocol on the substances that deplete the ozone layer was enforced in 1989 which banned the use of CFCs by the end of year 1995 in developed countries. This made way to the introduction of hydrofluorocarbons (HFCs) which have zero ozone depletion potential. HFCs matched the thermodynamic properties of CFCs [5-8], but showed poorer tribological performance in comparison to their predecessors [1]. The inability of HFCs to form surface films under normal compressor operating conditions 
contributed to their inferior tribological performance [2-4]. In addition mineral oils that showed good miscibility with CFCs and HCFCs, unfortunately, were not compatible with HFCs and synthetic oils with various additives had to be developed for HFCs [1]. Despite having these drawbacks, HFCs were globally accepted. The damaging implications of HFCs on the earth's atmosphere were realized much later when it was found that HFCs are one of the major contributors to global warming [9]. Kyoto Protocol to the United Nations Framework Convention on Climate Change in 1997 established binding limits on $\mathrm{CO}_{2}$ and other greenhouse gases, which included HFCs as well. HFCs are now in the process of being phased out and it is expected that by the end of year $2030 \mathrm{HFC}$ availability will drop by $79 \%$ in Europe.

Most of the naturally occurring compounds have either thermodynamic limitations or have high toxicity or high flammability. This means that new future generation of artificially formulated thermofluids have to be introduced. The challenge for the refrigerant manufacturers is even greater this time as they not only have to come up with refrigerants that have zero ozone depletion potential but also should have minimum global warming potential. The refrigerant manufacturers have introduced new thermofluids which have a lower environmental impact. Hydrofluoroolefins (HFOs) and hydrofluoroethers (HFEs) are the newly introduced replacement refrigerants which have been reported to have good heat transfer and thermodynamic properties [10-14]. However, no significant investigations have been published on the tribological performance of HFEs.

This work is being carried out to investigate the interfacial properties of HFEs, for this purpose an experimental setup has been designed and commissioned. The setup allows a platform to conduct detailed tribological testing under varying operating conditions.

\section{TEST RIG}

The test rig is based upon an existing reciprocating microfriction machine which has been modified to test the future generation of thermofluids. Bespoke design of the test rig is shown in Fig. 1. The most integral part of the rig is the testing chamber which houses the testing parts. The chamber is designed in a way to be fully sealed and hold pressure in a range of -1 to +3 bar. The moving components within the test chamber are sealed to the test chamber by means of steel bellows and Viton O-rings seals. The arrangement is such that all relative movement is accommodated by the bellows. O-ring contacts are all static, meaning that there are no sliding seals. The apparatus can be used to test separate contact configurations which include, point contact, line contact and area contact. For this study point contact configuration was chosen which allows maximum Hertzian contact pressure for the same applied load.

\subsection{Temperature monitoring and control}

A heater block is located at the bottom of the chamber. The heater block comprises electric heating elements possesing a heating power of $800 \mathrm{~W}$. The apparatus has been fitted with two K-type thermocouples. Thermocouple- 1 is directly embedded in the heater block that contains the electric heating elements. The heater block can be heated up to $200^{\circ} \mathrm{C}$. Thermocouple- 2 is a wire-type thermocouple that is positioned in the specimen cup, and it is fixed with the help of screws. The specimen cup contains the flat specimen and is filled with the thermofluid. Thermocouple- 2 directly measures the exact temperature of the thermofluid being tested and helps maintain the temperature of the tested thermofluid by utilizing feedback PID control during the course of a test. 


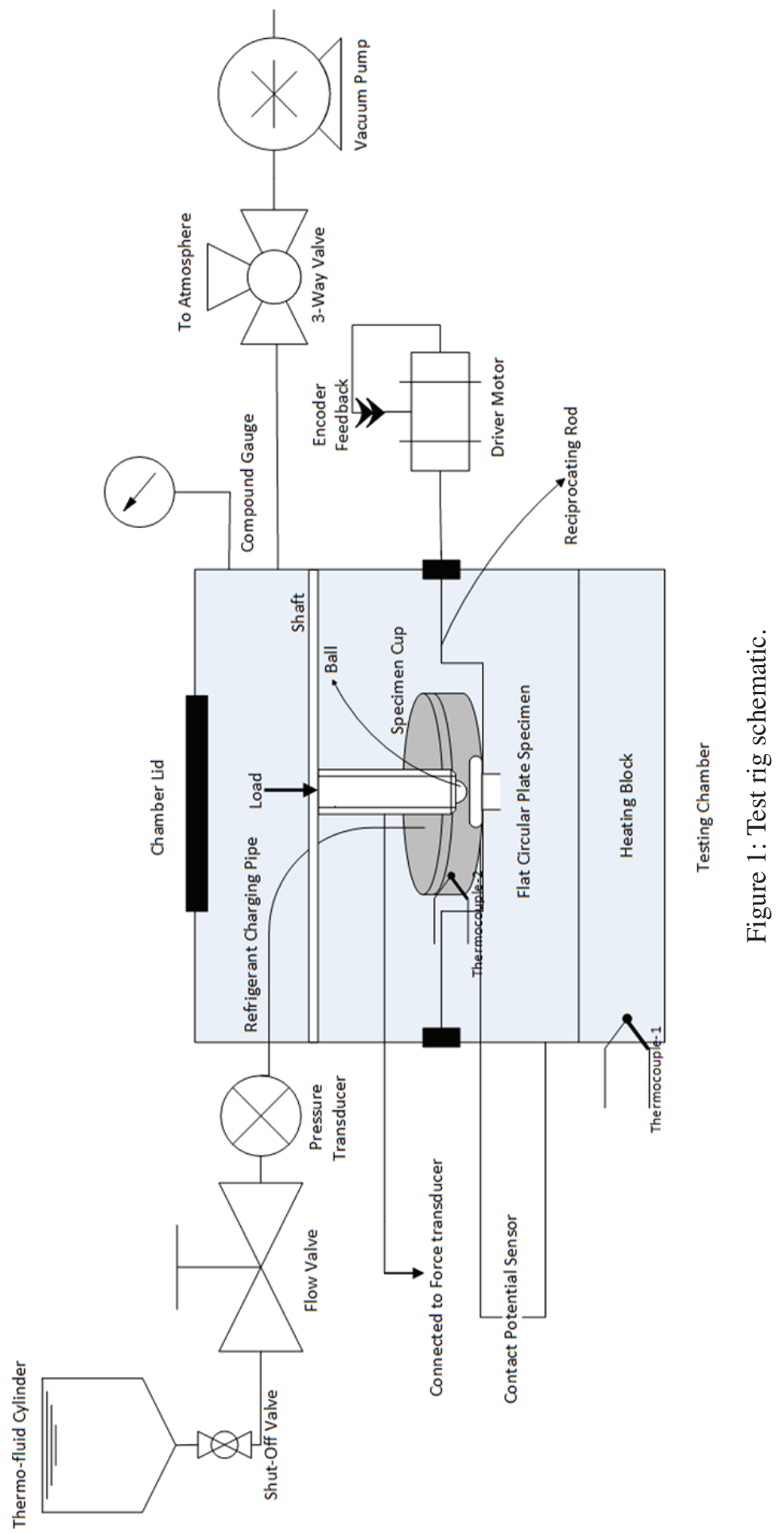




\subsection{Chamber pressure measurement}

The testing chamber can be pressurized up to +3 bar and can be vacuumed to -1 bar. A compound manual gauge and a pressure transducer have been installed to monitor both the positive and negative pressure in this range. The pressure transducer allows on screen motoring and recording of the chamber pressure during the course of an experiment. The pressure transducer is made of stainless steel having a transmitter range of -1 to +9 bar, it can measure the pressure of refrigerants in the range of -20 to $+120^{\circ} \mathrm{C}$. The output signal is linear in the range of 4 to $20 \mathrm{~mA}$, having a response time of less than $5 \mathrm{~ms}$. The pressure transducer is also shock and vibration resistant having good accuracy and repeatability. The compound gauge has been added for additional safety to always allow for visual pressure variations in the pressure chamber. If the data acquisition system or the computer freezes for some reason the manual gauge will always allow observations of the pressure readings to make sure that the pressure is within the safe operating limit.

\subsection{Lubricant film formation measurement}

Lubricant film formation between the contacts is sensed via a contact potential sensor. The contact potential measurement enables the observation of metal-to-metal interactions between the reciprocating parts. The data can be used to judge if a suitable lubrication film was formed during various testing conditions. The contact potential sensing is based on the principle of contact resistance measurement. The internal circuit for the contact potential sensor is shown in Fig. 2.

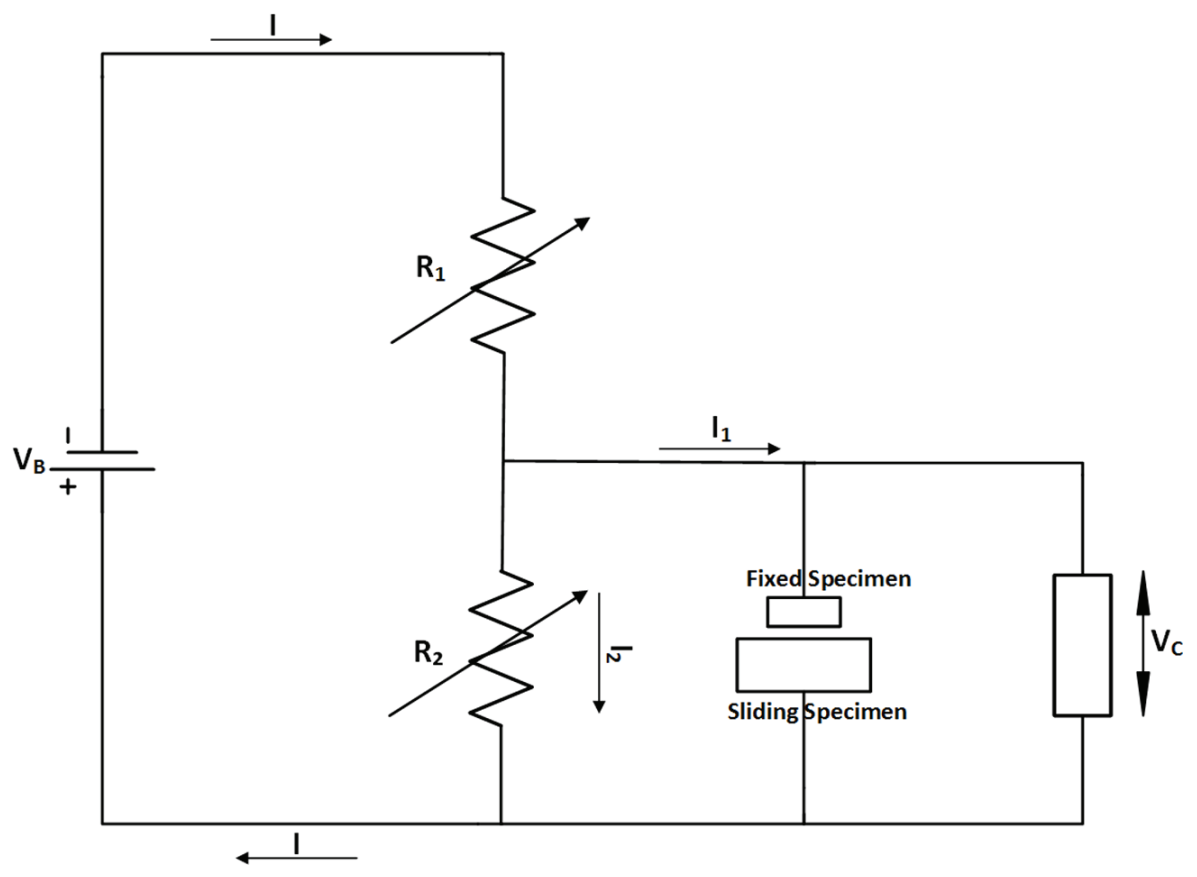

Figure 2: Contact potential measurement circuit. 
The internal electrical circuit allows the sensor to be calibrated in the range of $0-50 \mathrm{mV}$, with $0 \mathrm{~V}$ referring to short circuit (metal-to-metal contact) and $50 \mathrm{mV}$ means open circuit. The sensor has been fine-tuned with the help of the two variable resistors $R_{1}$ and $R_{2}$ to give values in the full range of $0-50 \mathrm{mV}$, and this increases the output resolution. $\mathrm{V}_{\mathrm{B}}$ is the excitation voltage applied to the circuit and $\mathrm{V}_{\mathrm{C}}$ is the output voltage that is sensed.

For the sensor to be installed, the two contact geometries have to be electrically isolated. If the specimens are not isolated before the start of an experiment then the sensor will always read the state of short circuit. The sensor should read a state of short circuit only when there is a metal-to-metal contact and should show values in the range of $0-50 \mathrm{mV}$ during testing. For this the two test specimens namely the flat circular plate and the steel ball have to be electrically isolated. The specimen holding cup has been isolated from the body of the machine using insulation tape and nylon screws. The specimen holding cup is used to hold the flat circular specimen, isolating the cup isolates the flat circular specimen from the steel ball.

\subsection{Friction force measurement}

The rig has been fitted with a Kistler Piezo-electric friction force transducer having a range of -100 to $100 \mathrm{~N}$. Normal load is applied by means of a spring balance attached to a cantilever arrangement. The cantilever exerts a normal force on the contact. The friction force sensor is attached to a yoke, and the yoke is connected to a force feedback rod upon which a housing is mounted that holds the fixed sample. The applied normal load and the values of the friction force are used to calculate the coefficient of friction in the software.

\subsection{Oscillating frequency measurement and control}

A $0.37 \mathrm{~kW}$ PID controlled DC motor drives the machine by sliding a lower flat plate sample in a reciprocating motion against a fixed upper sample using a scotch yoke mechanism. The mechanism is secured with the reciprocating rod which holds the flat plate specimen. The power is transmitted through the reciprocating rod which is supported by bearings on a linear guide rail. The setup allows the application of normal load in the range 5-50 N. The stroke length can be continuously varied between $1-5 \mathrm{~mm}$. The frequency of oscillation can be varied between $2.5-50 \mathrm{~Hz}$. A tachometer is coupled directly at the back end of the driver motor shaft which provides the rotational speed. The data from the tachometer is used as feedback to maintain a constant oscillating frequency with the help of PID control.

All the inputs and outputs are fed into a purpose built data acquisition system which serially transmits the data to a microprocessor. The computer has a specialized software that is used to monitor, control and record the operating parameters in real time.

\section{TESTING PROCEDURE}

Testing procedure consists of a number of different steps. 52100 steel ball bearings have been chosen as the ball specimen. Mild steel has been machined into flat circular disks and disks have been grinded and polished to an average surface roughness of $0.1 \mu \mathrm{m}$, which are used as the flat plate specimen. This arrangement allows ball-on-flat, that is, point contact configuration. The thermofluid being used in these tests is HFE-7000. HFE-7000 is a non-flammable, non-corrosive, low toxic chemical with a chemical formula $\mathrm{C}_{3} \mathrm{~F}_{7} \mathrm{OCH}_{3}$. It has a wide range of application areas which include use in high voltage transformer, in medical laboratory devices 
such as Histobath, in freeze-drying units, in ion implanters and in auto-cascade refrigeration systems. It has an ozone depletion potential of zero and a global warming potential value of 530 for 100-year integrated time horizon.

\subsection{Sample installation}

The flat circular specimen is secured in the cup on the oscillating rod with the help of screws. Then the wire type thermocouple- 2 is secured into position which is used to measure the thermofluid temperature. The ball is secured on the ball-holder with the help of grub screws and the ball holder is connected to a ball holder shaft. The shaft provides a means to fasten the ball-holder in position and also serves as a means to apply the vertical load. After this the chamber is closed and sealed.

\subsection{Thermofluid charging}

After closing the chamber it is vacuumed so as to minimize the effects of ambient air and oxygen during testing. After the chamber has been vacuumed the thermofluid is introduced in the system using the shut-off and flow valves. Sufficient amount of thermofluid is introduced in the cup so that the cup is full and the bottom flat specimen is fully immersed in the fluid to ensure fully lubricated conditions at all times. The vacuum inside the testing chamber and gravity helps the fluid to flow from the cylinder into the cup. The pressure inside the chamber is controlled in a way to keep the thermofluid in liquid state during testing to always maintain fully lubricated conditions. The chamber lid has a transparent glass top that allows a person to physically observe the chamber conditions at all times.

\subsection{Testing}

The desired load is manually applied. After this the control algorithm is run and the chamber thermofluid is heated to the desired temperature. The temperature of the fluid is precisely controlled using temperature values from thermocouple- 2 and feedback PID control. After the temperature has reached to its specific value, the temperature of the fluid is stabilized and maintained for $1 \mathrm{~h}$ before starting a test. After the temperature has been stabilized and has been maintained, a test is run for $2 \mathrm{~h}$. The oscillating frequency is precisely controlled using feedback controlled driver motor. The motor, the heater and all the transducers are connected to a microprocessor based central data acquisition and control system. The realtime values of the friction force, of the heater block temperature, temperature of the thermofluid, the chamber pressure and the motor speed are continuously recorded in a spreadsheet.

\section{RESULTS AND DISCUSSION}

The only published works on the tribological assessment of HFEs are [15, 16]. The study [15] was performed in 2002 which investigated the elastohydrodynamic and boundary lubrication performance of polyester (POE) in HFE-245mc and HFC-134a environment. A recent study [16] concerning the tribological performance of HFE-347mcc3 has been published in 2018. The study [16] however looks only into wear and there is no discussion about friction.

Tests in this study have been performed by varying the applied normal load and the thermofluid temperature. Each test was performed for $2 \mathrm{~h}$ and each test was repeated two times 
to ensure repeatability. The applied normal load was gradually increased from 10 to $30 \mathrm{~N}$ and the thermofluid temperature was increased from $20^{\circ} \mathrm{C}$ to $40^{\circ} \mathrm{C}$.

\subsection{Coefficient of friction}

The results for the average coefficient of friction are presented in Fig. 3. The average coefficient of friction at $10 \mathrm{~N}$ and $20^{\circ} \mathrm{C}$ thermofluid temperature is 0.44 , this value reduces to 0.32 at $10 \mathrm{~N}$, $40^{\circ} \mathrm{C}$. At $20 \mathrm{~N}, 20^{\circ} \mathrm{C}$ the average coefficient of friction is 0.28 which is reduced to 0.25 at $20 \mathrm{~N}$, $40^{\circ} \mathrm{C}$. At $30 \mathrm{~N}, 20^{\circ} \mathrm{C}$ the friction coefficient is 0.25 which decreases to 0.23 at $30 \mathrm{~N}, 40^{\circ} \mathrm{C}$.

For a given load increase in temperature decreases the coefficient of friction and the frictional force. The difference in reduction in the coefficient of friction is higher at lower loads. Increase in load at the same temperature also gives a lower coefficient of friction. With increasing load the coefficient of friction is reduced for the same thermofluid temperature but the difference in reduction is not as significant as at lower loads.

The coefficient of friction dropped due to the formation of $\mathrm{FeF}_{2}$ and iron oxide films after running-in of the components was achieved in the study [15] when POE was used in HFE$245 \mathrm{mc}$ environment, however no such tribo-films were detected when POE was tested with HFC-134a under identical testing conditions. The chemical composition of HFE-245mc is $\mathrm{CH}_{3} \mathrm{OCF}_{2} \mathrm{CF}_{3}$ which is similar to that of HFE-7000 with HFE-7000 having a larger molecular structure as compared to HFE-245mc. It is believed that similar to [15], the HFE in our case reacted with the rubbing surfaces as well and formed iron oxide and $\mathrm{FeF}_{2}$ films which reduced the coefficient of friction. The results show that increasing temperatures and operating loads will result in the reduction of the coefficient of friction. The decrease in coefficient of friction with increasing temperatures is due to the increase in reactivity of HFE-7000 with the interacting metals. Similarly increasing load at the same temperature decreased the coefficient of friction because increasing the load helped in the chemical breakdown and reaction of HFE-7000 with the interacting metals.

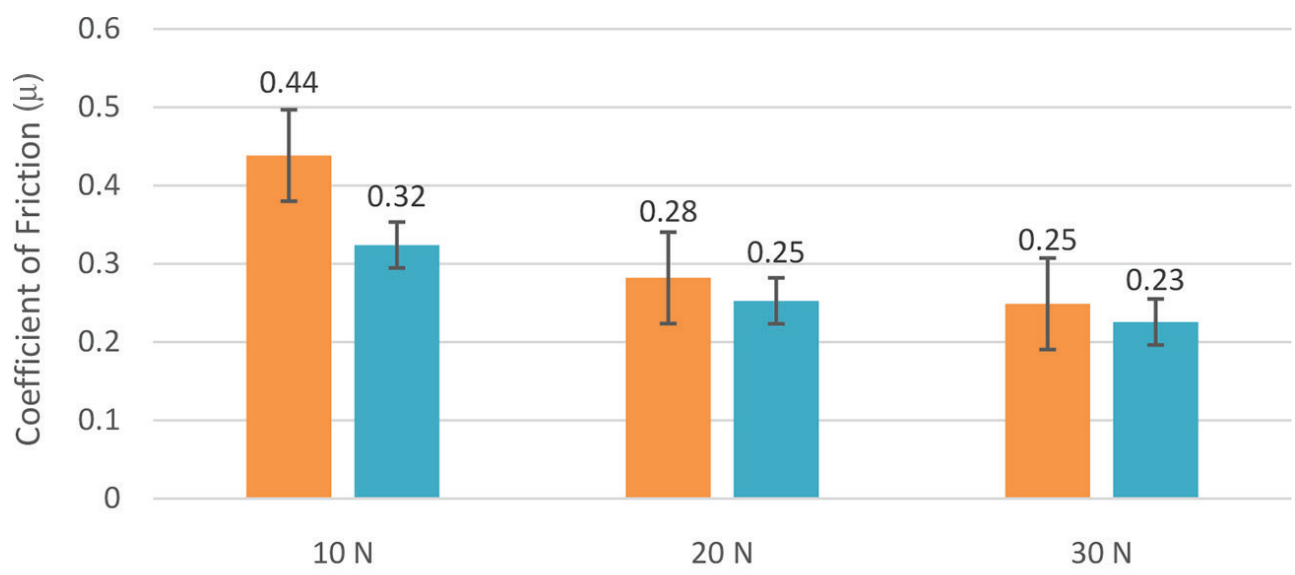

Normal Load, N

$20^{\circ} \mathrm{C}-40^{\circ} \mathrm{C}$

Figure 3: Effect of load and temperature on the average coefficient of friction. 


\subsection{Wear}

The wear track formed on the flat circular mild steel specimens was examined under a white light interferometer. The interferometer was used to 3D stitch the wear track which was then used to calculate the wear volume. $3 \mathrm{D}$ oblique plots for $20^{\circ} \mathrm{C}$ and $40^{\circ} \mathrm{C}$ testing conditions are shown in Figs. 4 and 5, respectively.

For the tests conducted at $20^{\circ} \mathrm{C}$, increasing the load results in a deeper and wider wear track. The widening of the wear track is associated with the change in sliding contact area of the point contact with time. As the testing progresses the point contact becomes flatter with wear which produces a wider wear track for each load. Increasing load produces a wider wear track due to the increased flattening on the ball. Increasing load also generates a deeper wear track. This shows that increasing load at the same temperature will produce more wear.

For the tests conducted at $40^{\circ} \mathrm{C}$, increasing load from 10 to $20 \mathrm{~N}$ generated a shallower and narrower wear track. Increasing the load further to $30 \mathrm{~N}$, produced the deepest and widest wear track for HFE-7000 temperature of $40^{\circ} \mathrm{C}$. This indicates that there exist an optimum load and temperature combination which produces the least amount of wear.

Wear volume was measured using the white light interferometer after obtaining the 3D profiles. The results of the wear volume have been summarized in Fig. 6. It is evident from Fig. 6 that increasing the load at $20^{\circ} \mathrm{C}$ thermofluid temperature generates more wear. It can also be clearly seen from Fig. 6 that increasing the testing temperature at the same load helps in reducing wear. The results of wear at $40^{\circ} \mathrm{C}$ however are different from the results of wear at $20^{\circ} \mathrm{C}$. Least amount of wear was obtained at $20 \mathrm{~N}, 40^{\circ} \mathrm{C}$ form all the samples tested.

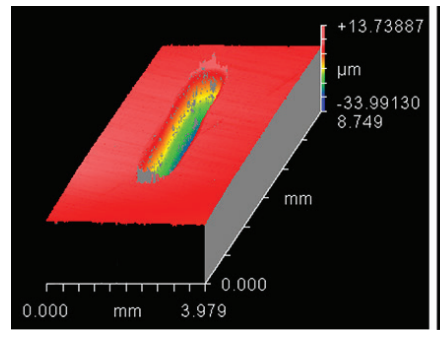

(a)

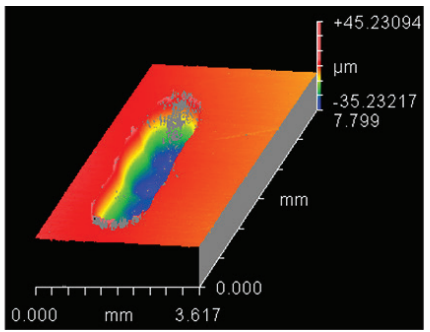

(b)

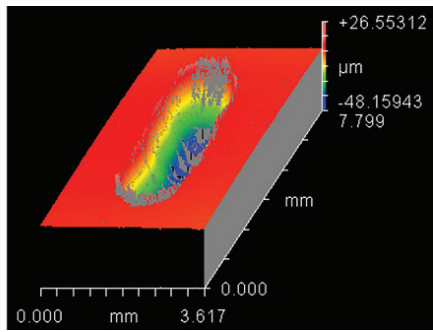

(c)

Figure 4: Wear track for HFE-7000 temperature $20^{\circ} \mathrm{C}$. At normal loads of: (a) $10 \mathrm{~N}$, (b) $20 \mathrm{~N}$ and (c) $30 \mathrm{~N}$.

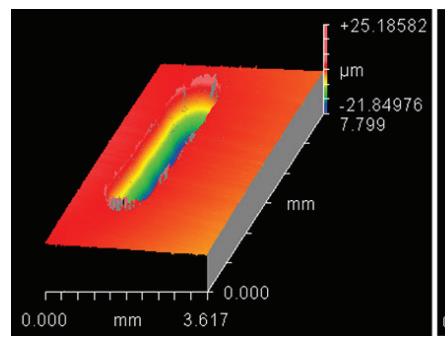

(a)

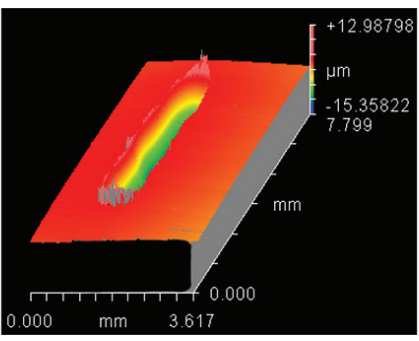

(b)

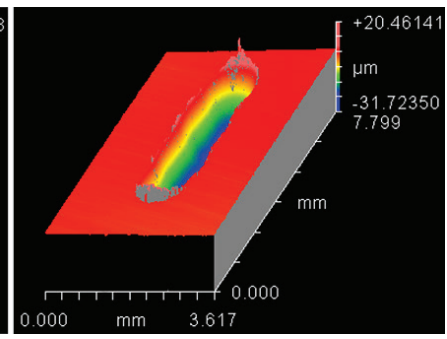

(c)

Figure 5: Wear track for HFE-7000 temperature $40^{\circ} \mathrm{C}$. At normal loads of: (a) $10 \mathrm{~N}$, (b) $20 \mathrm{~N}$ and (c) $30 \mathrm{~N}$. 


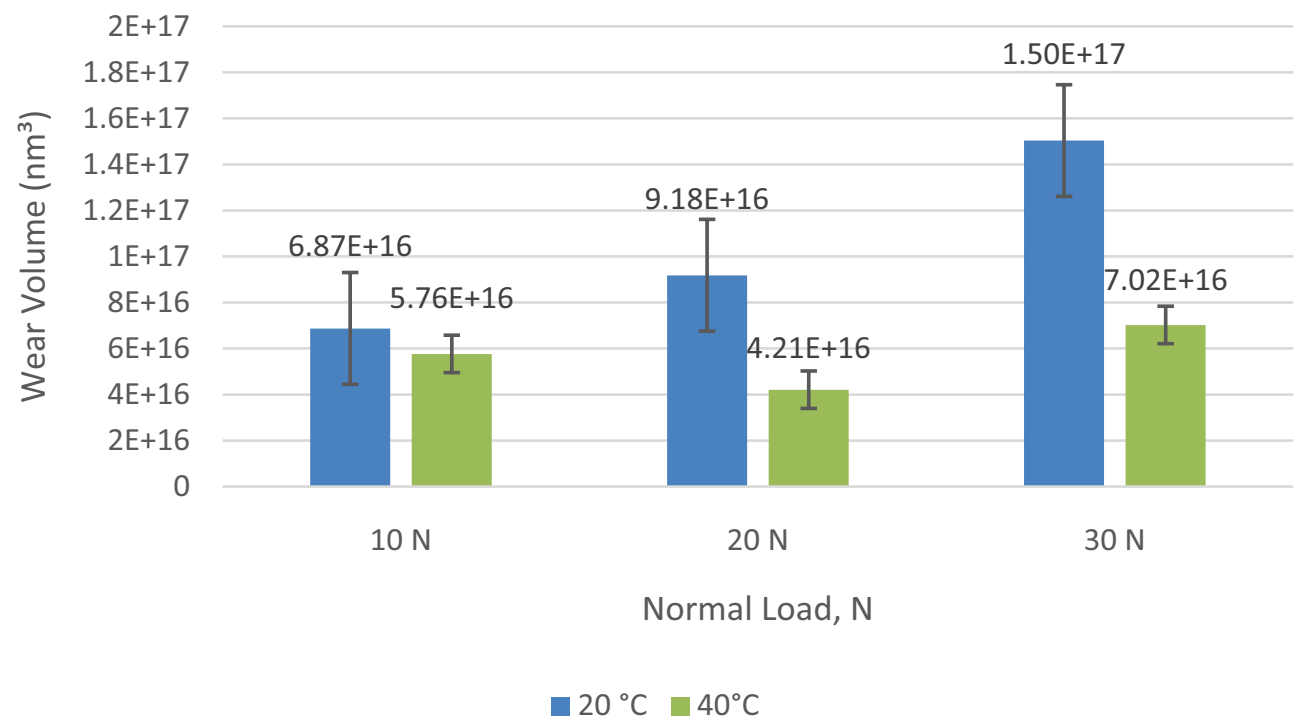

Figure 6: Effect of load and temperature on wear [16].

\section{CONCLUSION}

A micro friction testing machine has been successfully modified and commissioned that is capable of testing the next generation of thermofluids from a tribological view point. Various tests have been carried out by altering the testing conditions and the results for varying the applied normal load and by changing the operating temperature of HFE-7000 have been presented.

The results show that increasing the operating temperature and load reduces the coefficient of friction which is believed to be associated with the formation of protective tribo-films on the rubbing surfaces. The formation of these films is accelerated by increasing the temperature and applied load.

Wear volume increases with increasing load for $20^{\circ} \mathrm{C}$ thermofluid temperature. However wear volume decreases as the load is increased from $10 \mathrm{~N}$ to $20 \mathrm{~N}$ at thermofluid temperature of $40^{\circ} \mathrm{C}$. Upon increasing the applied normal load further from $20 \mathrm{~N}$ to $30 \mathrm{~N}$ at $40^{\circ} \mathrm{C}$ increases wear. This shows that there exists an optimum combination of load and temperature which generates the least amount of wear.

This study shows that HFE-7000 which is a promising new generation environmentally friendly thermofluid from a thermodynamics point of view also has good tribological properties. This study indicates that HFE-7000 is capable of generating protective tribo-films on the materials tested, which help reduce friction and wear. With these results it can be inferred that HFE-7000 based systems will offer better tribological performance in comparison to systems using HFCs.

\section{REFERENCES}

[1] Bhutta, M.U., Khan, Z.A., Garland, N.P. \& Ghafoor, A., A Historical Review on the Tribological Performance of Refrigerants used in Compressors. Tribology in Industry, 40(1), pp. 19-51, 2018, https://doi.org/10.24874/ti.2018.40.01.03

[2] Davis, B., Sheiretov, T. \& Cusano, C., Tribological Evaluation of Contacts Lubricated by Oil-Refrigerant Mixtures. Air Conditioning and Refrigeration Center, College of Engineering, University of Illinois at Urbana-Champaign, ACRC Technical Report, 19 May 1992. 
[3] Mizuhara, K., Akei, M. \& Matsuzaki, T., The friction and wear behavior in controlled alternative refrigerant atmosphere. Tribology Transactions, 37(1), pp. 120-128, 1994, https://doi.org/10.1080/10402009408983274

[4] Kawahara, K., Mishina, S., Kamino, A., Ochiai, K., Okawa, T. \& Fujimoto, S., Tribological Evaluation of Rotary Compressor with HFC Refrigerants, In International Compressor Engineering Conference, Purdue University, Indiana, USA, paper 1141, 23-26 July 1996.

[5] Spauschus, H.O., HFC 134a as a substitute refrigerant for CFC 12. International Journal of Refrigeration, 11(6), pp. 389-392, 1988, https://doi.org/10.1016/01407007(88)90063-1

[6] Shankland, I.R., Basu, R.S. \& Wilson, D.P., Thermal conductivity and viscosity of a new stratospherically safe refrigerant-1, 1, 1, 2-tetrafluoroethane (R-134a). In International Refrigeration and Air Conditioning Conference, Purdue University, Indiana, USA, paper 41, 1988.

[7] Eckels, S.J. \& Pate, M.B., An experimental comparison of evaporation and condensation heat transfer coefficients for HFC-134a and CFC-12. International Journal of Refrigeration, 14(2), pp. 70-77, 1991, https://doi.org/10.1016/0140-7007(91)90078-u

[8] Khan, S.H. \& Zubair, S.M., Thermodynamic analyses of the CFC-12 and HFC-134a refrigeration cycles. Energy, 18(7), pp. 717-726, 1993, https://doi.org/10.1016/03605442(93)90031-8

[9] Breidenich, C., Magraw, D., Rowley, A. \& Rubin, J.W., The Kyoto protocol to the United Nations framework convention on climate change. American Journal of International Law, 92(2), pp. 315-331, 1998, https://doi.org/10.2307/2998044

[10] Spatz, M., Minor, B. \& DuPont, H., HFO-1234yf A low GWP refrigerant for MAC. Honeywell/DuPont joint collaboration, In SAE World Congress, Detroit, Michigan, USA, 14-17 April 2008.

[11] Akasaka, R., An application of the extended corresponding states model to thermodynamic property calculations for trans-1,3,3,3-tetrafluoropropene (HFO-1234ze(E)). International Journal of Refrigeration, 33(5), pp. 907-914, 2010, https://doi. org/10.1016/j.ijrefrig.2010.03.003

[12] Richter, M., McLinden, M.O. \& Lemmon, E.W., Thermodynamic Properties of 2,3,3,3-Tetrafluoroprop-1-ene (R1234yf): Vapor Pressure and $\mathrm{p}-\rho-\mathrm{T}$ Measurements and an Equation of State. Journal of Chemical \& Engineering Data, 56(7), pp. 32543264, 2011, https://doi.org/10.1021/je200369m

[13] Helvaci, H. \& Khan, Z.A., Experimental study of thermodynamic assessment of a small scale solar thermal system. Energy Conversion and Management, 117, pp. 567-576, 2016, https://doi.org/10.1016/j.enconman.2016.03.050

[14] Helvaci, H.U. \& Khan, Z., Thermodynamic modelling and analysis of a solar organic Rankine cycle employing thermofluids. Energy Conversion and Management, 138, pp. 493-510, 2017, https://doi.org/10.1016/j.enconman.2017.02.011

[15] Muraki, M., Sano, T. \& Dong, D., Elastohydrodynamic properties and boundary lubrication performance of polyolester in a hydrofluoroether refrigerant environment, Proceedings of the Institution of Mechanical Engineers, Part J: Journal of Engineering Tribology, 216(1), pp. 19-26, 2002, https://doi.org/10.1243/1350650021543852

[16] Bhutta, M., Khan, Z. \& Garland, N., Wear performance analysis of Ni-Al2O3 nanocomposite coatings under nonconventional lubrication. Materials, 12(1), p. 36, 2018. doi: 10.3390/ma12010036 